\title{
Clinical significance of soluble CD163 in polymyositis-related or dermatomyositis- related interstitial lung disease
}

Yasunori Enomoto ${ }^{1,2}$, Yuzo Suzuki $^{1 *}$, Hironao Hozumi ${ }^{1}$, Kazutaka Mori $^{1}$, Masato Kono ${ }^{1}$, Masato Karayama ${ }^{1}$, Kazuki Furuhashi', Tomoyuki Fujisawa' ${ }^{1}$, Noriyuki Enomoto ${ }^{1}$, Yutaro Nakamura ${ }^{1}$, Naoki Inui ${ }^{1,3}$, Daisuke Suzuki ${ }^{4}$, Noriyoshi Ogawa ${ }^{4}$, Ran Nakashima ${ }^{5}$, Tsuneyo Mimori ${ }^{5}$, Toshihide Iwashita ${ }^{2}$ and Takafumi Suda ${ }^{1}$

\begin{abstract}
Background: Macrophage activation is involved in the pathogenesis of polymyositis (PM)/dermatomyositis (DM). CD163, a scavenger receptor expressed on the surface of activated macrophages, mediates anti-inflammatory functions. This study aimed to evaluate the clinical significance of soluble CD163 (sCD163) in PM/DM-related interstitial lung disease (ILD).

Methods: The main subjects were 48 patients with PM/DM-related ILD. As controls, 10 patients with PM/DM without ILD and 20 healthy volunteers were enrolled. In patients with PM/DM-related ILD, the baseline characteristics and clinical course were obtained through a review of patient medical records. Serum sCD163 levels at ILD diagnosis were quantified by enzyme-linked immunosorbent assay, which were compared with the other baseline clinical factors and evaluated for potential as a prognostic biomarker. In addition, immunohistochemistry analysis using anti-human CD163 antibody was performed on the lung sections of two patients with DM-related ILD (a survivor and non-survivor, respectively) and one patient with early-stage lung cancer as a normal control.

Results: The median value of serum SCD163 in patients with PM/DM-related ILD was $818 \mathrm{ng} / \mathrm{mL}$, which was higher than that of PM/DM patients without ILD and healthy volunteers $(716 \mathrm{ng} / \mathrm{mL}$ and $340 \mathrm{ng} / \mathrm{mL}$, respectively). Significant but mild correlations with serum sCD163 levels were observed for serum C-reactive protein levels $(r=0.322)$ and $\%$ predicted forced vital capacity $(r=-0.301)$ in patients with PM/DM-related ILD. A Cox proportional hazard model demonstrated that patients with PM/DM-related ILD and higher SCD163 levels had worse prognosis (age-adjusted and gender-adjusted hazard ratio per $100 \mathrm{ng} / \mathrm{mL}$ increase 1.27, 95\% confidence interval 1.11-1.45, $P<0.001)$. In immunohistochemistry analysis, compared with normal lung, alveolar infiltration of CD163-positive macrophages was evident in the lungs of patients with DM-related ILD. Especially, the finding was more severe in the non-survivor's lung.
\end{abstract}

Conclusions: Serum sCD163 might be a potential biomarker for predicting the severity and prognosis of PM/DMrelated ILD. Our results suggest the importance of macrophage activation in the disease.

Keywords: CD163, Myositis, Interstitial lung diseases, Macrophage, Biomarker

\footnotetext{
* Correspondence: yuzosuzu@hama-med.ac.jp

'Second Division, Department of Internal Medicine, Hamamatsu University

School of Medicine, 1-20-1 Handayama, Hamamatsu, Shizuoka 431-3192,

Japan

Full list of author information is available at the end of the article
} 


\section{Background}

Polymyositis (PM) and dermatomyositis (DM) are systemic inflammatory diseases affecting the muscles, skin, and several other organs including the lungs $[1,2]$. Interstitial lung disease (ILD) is a common complication seen in $20-78 \%$ of patients with PM/DM and can be a prognostic determinant [3-8]. Particularly, patients with DM and clinically amyopathic DM (CADM) frequently develop an acute and severe form of ILD [4, 5]. Thus, management of ILD is essential for the care of patients with $\mathrm{PM} / \mathrm{DM}$, and appropriate biomarkers reflecting the disease severity are required.

CD163, a scavenger receptor specifically expressed on the surface of activated macrophages and monocytes, is involved in the uptake of hemoglobin-haptoglobin and mediates the clearance of hemoglobin [9]. CD163 expression is upregulated by several inflammatory cytokines including interleukin (IL)-6 [10, 11]. Inflammatory infiltrates containing CD163-positive macrophages are frequently found in the diseased muscles in PM/DM [12, 13], where activated macrophages themselves directly induce inflammation and also mediate inflammatory responses via lymphocyte activation [14, 15]. Indeed, PM/ DM is one of the diseases that occasionally cause macrophage activation syndrome (MAS). In contrast, macrophages could produce transforming growth factor- $\beta$, the most prominent fibrosis-inducing molecule, in chronic lung inflammation [16]. Additionally, activated macrophages expressing CD163 have been demonstrated to play a key role during lung fibrosis [17]. Macrophage activation would be involved in the pathogenesis of PM/ DM-related ILD.

Upon acute and chronic inflammations, CD163 is shed from surface of activated macrophages and released into peripheral blood in the soluble form [11]. Therefore, soluble CD163 (sCD163) in the serum has been highlighted as a representative of macrophage activation and as a biomarker in several inflammatory diseases [18-23]. However, the clinical significance of this molecule in PM/DM-related ILD is not fully understood. The aim of this retrospective study was to evaluate the potential of serum sCD163 as a biomarker in PM/DM-related ILD.

\section{Methods}

\section{Patient selection}

A retrospective review of 52 patients with PM/DM-related ILD, consecutively admitted to Hamamatsu University Hospital between 1995 and 2014, was conducted. Subsequently, four patients (one with PM, one with CADM, and two with classic DM) who received immunosuppressive treatment with corticosteroids on diagnosis of ILD were excluded. Thus, this study included the remaining 48 patients with PM/DM-related ILD. Among them, 45 patients had never received immunosuppressive treatment, whereas the other 3 patients had received treatment but terminated it at least 1 year before the ILD diagnosis. In addition, 10 patients with PM/DM without ILD, who had not received immunosuppressive treatment, and 20 age-matched and gender-matched healthy volunteers were enrolled as control groups (Additional file 1: Table S1).

Classic PM/DM was diagnosed according to the criteria of Bohan and Peter [2]. Patients with definite or probable $\mathrm{PM} / \mathrm{DM}$ according to these criteria were included in the study. CADM was diagnosed when a patient had a skin rash typical of DM without clinical evidence of myositis and with little or no increase in serum creatine kinase $(\mathrm{CK})[5,24]$. The diagnosis of classic PM/DM and CADM had been confirmed by the dermatologists and/or rheumatologists and the pulmonologists in our institution. The diagnosis of ILD was based on the radiological assessment of high-resolution computed tomography (HRCT) of the chest, which included appearances of bilateral reticulation, ground-glass attenuation, or consolidation.

\section{Data collection}

The patients' clinical data at the time of PM/DM-related ILD diagnosis were obtained through medical record reviews. These data included symptoms; laboratory findings; pulmonary function test results (forced vital capacity (FVC); diffusing capacity of the lung for carbon monoxide (DLco), measured using a single breath technique); analyses of bronchoalveolar lavage (BAL) fluid; treatment; and prognosis.

Serum sCD163 levels were quantified by enzymelinked immunosorbent assay in accordance with the manufacturer's protocol (eBioscience, USA). The samples were obtained at the time of PM/DM-related ILD diagnosis and stored at $-80{ }^{\circ} \mathrm{C}$ for future analysis. All measurements were done in duplicate and the mean value of serum sCD163 was recorded. Written informed consent for serum conservation was obtained from all the subjects.

\section{Immunohistochemical analysis}

Lung specimens were obtained from two patients with classic DM-related ILD (on surgical lung biopsy and autopsy, respectively) and one patient with early-stage lung cancer as a normal control (on lung resection). These specimens had been fixed in $10 \%$ formalin and embedded in paraffin. Deparaffinized sections $(5 \mu \mathrm{m}$ thick) were preheated to $120{ }^{\circ} \mathrm{C}$ for 20 minutes. After blocking endogenous peroxidase activity with $0.3 \% \mathrm{H}_{2} \mathrm{O}_{2}$ for 20 minutes, the slides were incubated with the rabbit anti-human CD163 monoclonal antibody (1:200; Leica Biosystems, Germany) or isotype control antibody (Jackson ImmunoResearch Laboratories, USA) for $1 \mathrm{~h}$ at 
$20{ }^{\circ} \mathrm{C}$. Subsequently, the sections were incubated with biotin-conjugated gout anti-rabbit IgG antibody (Nichirei, Japan) for 15 minutes, and the immunoreaction was visualized using a 3,3-diaminobenzidine (DAB) chromogen solution (DAB substrate kit; Vector Laboratories, USA), and then counterstained with hematoxylin. Additionally, hematoxylin-eosin staining was also done on another slide from each patient.

\section{Statistical analysis}

Data were described as numbers (percentages) or medians with observed ranges. Spearman's rank correlation coefficients were evaluated for correlation between serum sCD163 and the other clinical factors with continuous variables. The Mann-Whitney $U$ test was performed for two-group comparisons. One-way analysis of variance was performed followed by post-hoc analysis (Dunn's multiple comparisons test) for multi-group comparisons. Overall survival time was defined as the number of months from PM/DM-related ILD diagnosis (the date of the first HRCT evaluation) until all-cause death or at censoring. Patients were censored if alive on 30 June 2015. Cox proportional hazard modeling was used to identify prognostic factors. To estimate the cutoff value of serum sCD163 level, time-dependent receiver operating characteristic analysis was performed, as described previously $[25,26]$. The Kaplan-Meier survival method and the log-rank test were employed to compare the survival rate between groups. Values of $P<$ 0.05 were considered significant. Statistical analyses were performed by using $\mathrm{R}$ software version 2.15.1 (The $\mathrm{R}$ Foundation for Statistical Computing, Austria) and SPSS software version 13.0 (SPSS, USA).

\section{Results}

\section{Baseline characteristics}

Baseline characteristics of patients with PM/DM-related ILD are summarized in Table 1. The numbers of patients diagnosed with classic DM, CADM, and PM were 24, 21 , and 3 , respectively. Twenty patients (42\%) had a smoking history. The median serum levels of CK and Creactive protein (CRP) were almost within normal limits. Serum ferritin levels were highly variable, ranging from $14 \mathrm{ng} / \mathrm{mL}$ to $12,701 \mathrm{ng} / \mathrm{mL}$. Using immunoprecipitation methods, anti-melanoma differentiation-associated genes 5 (MDA5) antibody and anti-aminoacyl-tRNA synthetase (ARS) antibody were detected in 14 patients (29\%) and 19 patients (40\%), respectively. Mildly decreased values of arterial partial pressure of oxygen $\left(\mathrm{PaO}_{2}\right)$, percent predicted FVC, and percent predicted DLco were commonly observed. The BAL fluid revealed a slightly high percentage of lymphocytes (median 7.2\%, range $1.2-70.0 \%)$. All patients received radiological examinations using chest HRCT, in which bilateral
Table 1 Baseline characteristics and the correlations with serum soluble CD163 levels in patients with polymyositis/dermatomyositisrelated interstitial lung disease

\begin{tabular}{|c|c|c|c|}
\hline Variables & Patients $(n=48)$ & $r$ & $P$ value \\
\hline $\begin{array}{l}\text { Age at the ILD diagnosis } \\
\text { (years) }\end{array}$ & $55,32-76$ & -0.05 & 0.72 \\
\hline Male & $17(35 \%)$ & - & - \\
\hline Current or former smoker & $20(42 \%)$ & - & - \\
\hline CADM/classic DM/PM & $21(44 \%) / 24(50 \%) / 3(6 \%)$ & - & - \\
\hline Fever & $20(42 \%)$ & - & - \\
\hline Cough & $20(42 \%)$ & - & - \\
\hline Dyspnea & $23(48 \%)$ & - & - \\
\hline Muscle pain or weakness & $27(56 \%)$ & - & - \\
\hline Rash typical of DM & $45(94 \%)$ & - & - \\
\hline Raynaud's phenomenon & $5(10 \%)$ & - & - \\
\hline Arthralgia & $17(35 \%)$ & - & - \\
\hline CK (IU/mL) & $162,24-5274$ & 0.24 & 0.10 \\
\hline CRP (mg/dL) & $0.36,0.03-8.33$ & 0.32 & 0.03 \\
\hline $\mathrm{KL}-6(\mathrm{U} / \mathrm{mL})$ & $883,249-4323$ & 0.14 & 0.33 \\
\hline Ferritin (ng/mL) & $116.5,14-12701$ & 0.25 & 0.08 \\
\hline $\mathrm{PaO}_{2}$ on room air (Torr) & $75.3,47.9-109.0$ & -0.16 & 0.28 \\
\hline $\begin{array}{l}\text { Anti-MDA5-positive/anti-ARS } \\
\text { positive/others }\end{array}$ & $\begin{array}{l}14(29 \%) / 19(40 \%) / 15 \\
(31 \%)\end{array}$ & - & - \\
\hline Predicted FVC (\%) & $65.8,40.6-107.7(n=45)$ & -0.30 & 0.045 \\
\hline Predicted DLco (\%) & $60.3,27.4-127.2(n=18)$ & -0.15 & 0.55 \\
\hline BAL-lymphocytes (\%) & $7.2,1.2-70.0(n=35)$ & 0.03 & 0.85 \\
\hline
\end{tabular}

Data are presented as number (\%) or median, range. All $P$ values are derived from the Spearman's rank correlation coefficient. ARS aminoacyl-tRNA synthetase, $B A L$ bronchoalveolar lavage, CADM clinically amyopathic dermatomyositis, $C K$ creatine kinase, CRP C-reactive protein, DLco diffusing capacity of the lung for carbon monoxide, DM dermatomyositis, FVC forced vital capacity, ILD interstitial lung disease, $K L-6$ Krebs von den Lungen-6, $\mathrm{PaO} 2$ arterial partial pressure of oxygen, MDA5 melanoma differentiation-associated gene 5 ,

$P M$, polymyositis

consolidation and/or ground glass attenuation were commonly shown.

\section{Serum sCD163}

Serum concentrations of $\mathrm{sCD} 163$ are compared in Fig. 1a. The median value of serum sCD163 was $818 \mathrm{ng} /$ $\mathrm{mL}$ (range 300-2759 $\mathrm{ng} / \mathrm{mL}$ ) in patients with PM/DMrelated ILD, which was relatively higher than that in patients with PM/DM without ILD (median $716 \mathrm{ng} / \mathrm{mL}$, range $250-1101 \mathrm{ng} / \mathrm{mL}$ ). Patients with PM/DM, regardless of the presence/absence of ILD, had significantly higher sCD163 levels than healthy volunteers (median $340 \mathrm{ng} / \mathrm{mL}$, range $127-647 \mathrm{ng} / \mathrm{mL}$ ).

Table 1 shows the correlation between serum sCD163 levels and other clinical factors at PM/DM-related ILD diagnosis. Although the coefficients were generally low, serum sCD163 was positively correlated with serum CRP $(r=0.322, \quad P=0.03)$ and negatively correlated with \% 

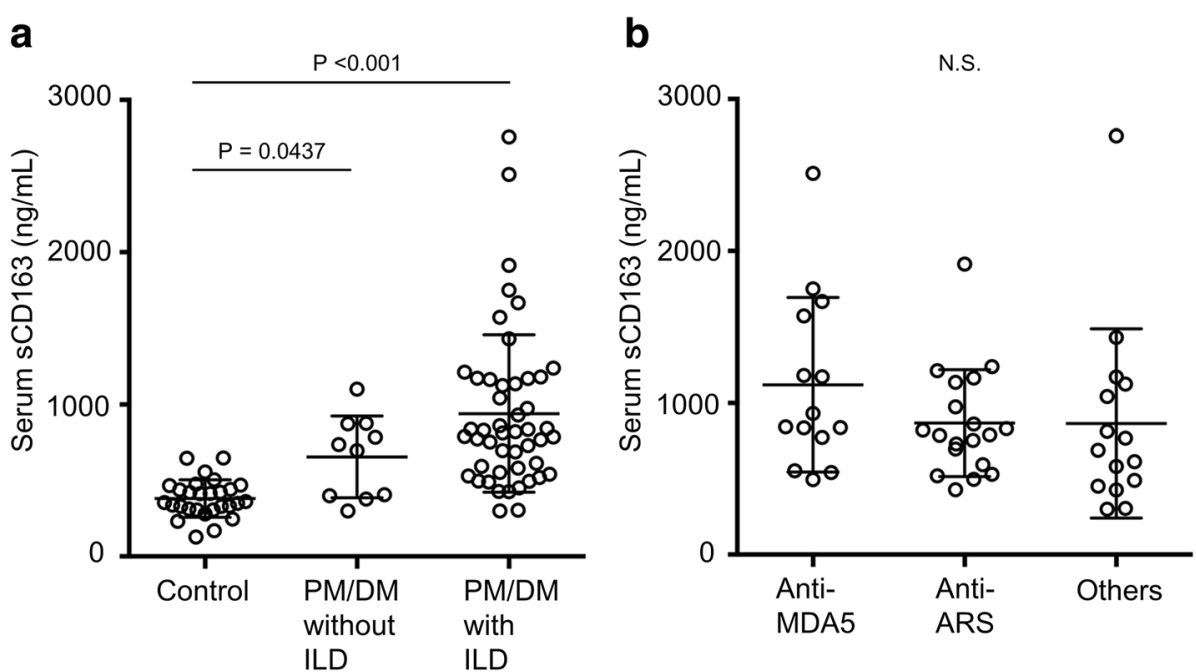

Fig. 1 Comparison of serum soluble CD163 levels. a Patients with polymyositis (PM)/dermatomyositis (DM)-related interstitial lung disease (ILD) $(n=48)$ vs patients with PM/DM without ILD $(n=10)$ vs healthy volunteers $(n=20)$. b Patients with PM/DM-related ILD with anti-melanoma differentiation-associated gene 5 (MDA5) antibody $(n=14)$ vs those with anti-aminoacyl-tRNA synthetase $(A R S)$ antibody $(n=19)$ vs those without both antibodies $(n=15)$. One-way analysis of variance followed by post-hoc analysis (Dunn's multiple comparisons test) was used for multi-group comparisons

predicted FVC $(r=-0.301, P=0.045)$. There was weak, non-significant correlation with serum ferritin $(r=$ $0.25, P=0.08)$. Patients with fever and arthralgia had significantly higher sCD163 than those without such symptoms (Table 2). The comparison of sCD163 levels according to the profile of myositis-specific autoantibodies is shown in Fig. 1b. Although not significant, patients with anti-MDA5 antibody exhibited relatively higher values.

\section{Clinical course}

The median follow-up period in patients with PM/DM-related ILD was 85.2 months (range 5.8-211.4 months). Only two patients were lost to follow up. Most patients (46/48, 96\%) received immunosuppressive therapy after ILD diagnosis. Among these patients, combination regimens of high-dose corticosteroids and immunosuppresssants, including cyclophosphamide, cyclosporine, and tacrolimus, were administered to 38 patients; in contrast, corticosteroid monotherapy was selected for the other 8 patients. Although most patients had a favorable response to the treatment, 13 patients died during the follow-up period. The most common cause of death was respiratory failure due to ILD $(n=9)$, followed by cancer of unknown primary origin $(n=1)$, myocarditis $(n=1)$, rupture of aortic aneurysm $(n=1)$, and unknown cause $(n=1)$. Among the non-surviving patients, lung specimens were available in three patients, which showed diffuse alveolar damage on surgical lung biopsy $(\mathrm{n}=1)$ or autopsy $(\mathrm{n}=1)$ and usual interstitial pneumonia with moderate lymphocytic alveolitis on surgical lung biopsy $(\mathrm{n}=1)$.
The results of the univariate Cox proportional hazard model are listed in Table 3. Among the baseline clinical factors, presence of fever, higher level of serum ferritin, higher level of serum sCD163, lower $\mathrm{PaO}_{2}$, and lower percent predicted FVC were significantly associated with worse prognosis. The age-adjusted and gender-adjusted hazard ratio for serum sCD163 (per $100 \mathrm{ng} / \mathrm{mL}$ increase) was 1.27 (95\% confidence interval 1.11-1.45, $P<0.001$ ). Additionally, even when classifying the four patients whose cause of death was not respiratory failure due to ILD into censored cases, the prognostic significance of serum sCD163 remained (age-adjusted and genderadjusted hazard ratio $1.26,95 \%$ confidence interval 1.09-1.45, $P=0.002$ ). As shown in Fig. 2a, the cutoff for survival was calculated as $800 \mathrm{ng} / \mathrm{mL}$. The comparisons of baseline characteristics between patients with higher and lower values of serum sCD163 are summarized in Additional file 1: Table S2. The patients with higher serum sCD163 had significantly lower survival rates than those with lower serum sCD163 (log rank test, $P=$ 0.043) (Fig. 2b).

\section{Immunohistochemical findings}

The immunohistochemical results for lung sections are shown in Fig. 3. CD163 expression in resected lung sections from an early lung cancer patient (Fig. 3a-d), a surviving patient with classic DM-related ILD (Fig. 3eh; 66 year-old male patient, serum sCD163 $=519 \mathrm{ng} /$ $\mathrm{mL}$ ), and a non-surviving patient with classic DMrelated ILD (Fig. 3i-l; 32 year-old male patient, serum $\mathrm{sCD} 163=1572 \mathrm{ng} / \mathrm{mL}$ ) were evaluated. Compared with 
Table 2 Comparison between baseline categorical variables and serum soluble CD163 levels in patients with polymyositis/ dermatomyositis-related interstitial lung disease

\begin{tabular}{|c|c|c|}
\hline Variables & Soluble CD163, ng/mL & $P$ value \\
\hline Male/female & $814(300-1572) / 822(305-2759)$ & 0.66 \\
\hline $\begin{array}{l}\text { Current or former } \\
\text { smoker/never smoker }\end{array}$ & $791(300-2758) / 834(305-2512)$ & 0.39 \\
\hline CADM/others & $730(305-1750) / 862(300-2759)$ & 0.09 \\
\hline Fever, yes/no & $1009(428-2512) / 742(300-2759)$ & 0.03 \\
\hline Cough, yes/no & $806(426-1572) / 826$ (300-2759) & 0.59 \\
\hline Dyspnea, yes/no & $814(428-2759) / 822(300-1572)$ & 0.48 \\
\hline $\begin{array}{l}\text { Muscle pain or weakness, } \\
\text { yes/no }\end{array}$ & $862(300-2759) / 730(305-1750)$ & 0.09 \\
\hline Rash typical of DM, yes/no & $789(300-2759) / 1124(814-1213)$ & 0.30 \\
\hline $\begin{array}{l}\text { Raynaud's phenomenon, } \\
\text { yes/no }\end{array}$ & $593(426-1137) / 822(300-2759)$ & 0.27 \\
\hline Arthralgia, yes/no & $1124(305-2512) / 773(300-2759)$ & 0.02 \\
\hline $\begin{array}{l}\text { Anti-MDA5 antibody, } \\
\text { positive/negative }\end{array}$ & $887(495-2512) / 778(300-2759)$ & 0.09 \\
\hline
\end{tabular}

Data are presented as median (observed range). All $P$ values are derived from the Mann-Whitney $U$ test. CADM clinically amyopathic dermatomyositis, $D M$ dermatomyositis, MDA5 Melanoma differentiation-associated gene 5 normal lung, accumulation of CD163-positive macrophages at alveolar spaces was more evident in the lungs of the two patients with classic DM-related ILD. Especially, the macrophage infiltration in the non-survivor's lung was apparently severe, mainly at alveolar spaces and partly at alveolar walls.

\section{Discussion}

In this study, we evaluated the clinical significance of sCD163 in patients with PM/DM-related ILD. Serum sCD163 levels at ILD diagnosis were associated with several clinical factors and even the prognosis, suggesting that this molecule might be a potential biomarker of the disease.

The expression of CD163, a macrophage-specific marker within the macrophage/monocyte lineage, is intricately regulated by various factors, such as IL-6, IL-10, interferon- $\gamma$, tumor necrosis factor (TNF) $-\alpha$, and corticosteroids. Cleavage of CD163 from activated macrophages, which is performed by a disintegrin and metalloproteinase $17 / \mathrm{TNF}-\alpha$ converting enzyme in response to several inflammatory mediators, leads to an increase in sCD163 in the serum $[10,11]$. In fact, the serum sCD163 levels in our patients were correlated with the presence of fever and serum CRP levels,

Table 3 Analysis of prognostic factors in patients with polymyositis/dermatomyositis-related interstitial lung disease using the Cox proportional hazard model

\begin{tabular}{|c|c|c|c|c|c|c|}
\hline Variables & Unadjusted HR & $95 \% \mathrm{Cl}$ & $P$ & $\begin{array}{l}\text { Age-adjusted and } \\
\text { gender-adjusted HR }\end{array}$ & $95 \% \mathrm{Cl}$ & $P$ \\
\hline Age (per 1 year increase) & 1.01 & $0.96-1.07$ & 0.62 & - & - & - \\
\hline Male & 1.93 & $0.61-6.09$ & 0.26 & - & - & - \\
\hline Current or former smoker & 2.27 & $0.71-7.23$ & 0.17 & 1.96 & $0.47-8.24$ & 0.36 \\
\hline CADM & 0.37 & $0.10-1.38$ & 0.14 & 0.41 & $0.11-1.60$ & 0.12 \\
\hline Fever & 4.29 & $1.27-14.50$ & 0.02 & 4.68 & $1.36-16.10$ & 0.01 \\
\hline Cough & 0.65 & $0.20-2.16$ & 0.48 & 0.76 & $0.22-2.67$ & 0.67 \\
\hline Dyspnea & 2.30 & $0.69-7.64$ & 0.18 & 2.65 & $0.78-9.03$ & 0.12 \\
\hline Muscle pain or weakness & 2.69 & $0.73-9.97$ & 0.14 & 2.44 & $0.63-9.48$ & 0.20 \\
\hline Rash typical for DM & 0.40 & $0.09-1.85$ & 0.24 & 0.47 & $0.10-2.27$ & 0.35 \\
\hline Raynaud's phenomenon & 2.99 & $0.81-11.13$ & 0.10 & 3.03 & $0.72-12.84$ & 0.13 \\
\hline Arthralgia & 1.54 & $0.49-4.90$ & 0.46 & 1.39 & $0.42-4.59$ & 0.59 \\
\hline CK (per $10 \mathrm{IU} / \mathrm{mL}$ increase) & 1.00 & $0.995-1.01$ & 0.97 & 1.00 & $0.994-1.006$ & 0.96 \\
\hline CRP (per 1 mg/dL increase) & 1.13 & $0.91-1.41$ & 0.27 & 1.13 & $0.91-1.41$ & 0.27 \\
\hline KL-6 (per 100 U/mL increase) & 1.06 & $0.998-1.12$ & 0.06 & 1.05 & $0.995-1.12$ & 0.07 \\
\hline Ferritin (per 100 ng/mL increase) & 1.02 & $1.01-1.04$ & 0.01 & 1.03 & $1.01-1.05$ & 0.01 \\
\hline Soluble CD163 (per 100 ng/mL increase) & 1.22 & $1.09-1.36$ & 0.001 & 1.27 & $1.11-1.45$ & $<0.001$ \\
\hline $\mathrm{PaO}_{2}$ on room air (per 10 Torr increase) & 0.48 & $0.30-0.76$ & 0.002 & 0.48 & $0.28-0.81$ & 0.01 \\
\hline Anti-MDA5-positive & 2.60 & $0.81-8.32$ & 0.11 & 3.11 & $0.90-10.74$ & 0.07 \\
\hline Percent predicted FVC (per 10\% increase) & 0.73 & $0.61-0.88$ & 0.001 & 0.65 & $0.50-0.83$ & 0.001 \\
\hline
\end{tabular}

ARS aminoacyl-tRNA synthetase, CADM clinically amyopathic dermatomyositis, Cl confidence interval, $C K$ creatine kinase, CRP C-reactive protein, DM dermatomyositis, FVC forced vital capacity, HR hazard ratio, KL-6 Krebs von den Lungen-6, MDA5 melanoma differentiation-associated gene 5 

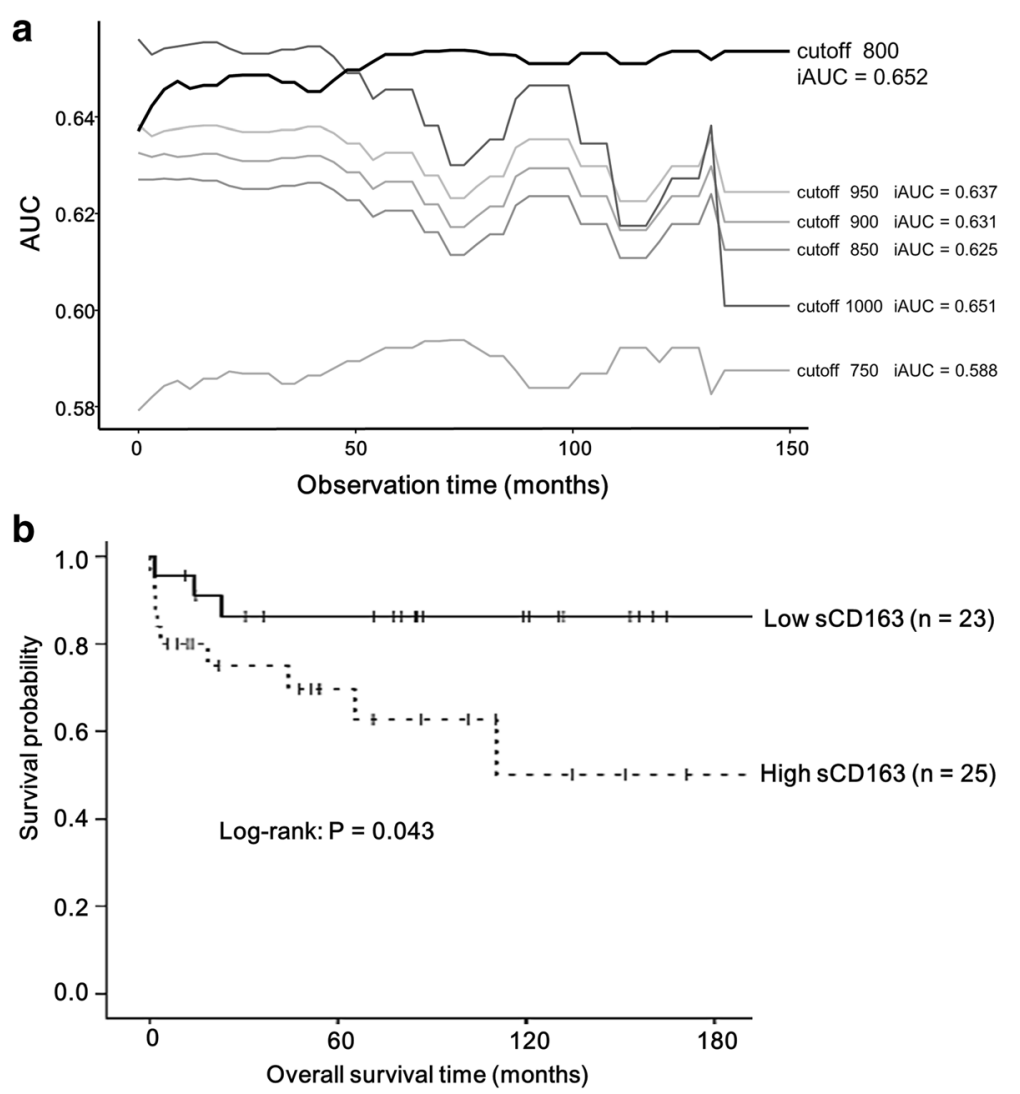

Fig. 2 Prognostic analysis. a Estimation of the cutoff for serum soluble CD163. b Survival curves in patients with polymyositis/dermatomyositisrelated interstitial lung disease. The cutoff for serum soluble CD163 is set at $800 \mathrm{ng} / \mathrm{mL}$ by comparing the integrated area under the curve (iAUC)

suggesting that elevated serum sCD163 is associated with systemic inflammation. Notably, among macrophages, CD163 is mainly expressed on alternatively activated macrophages, rather than classically activated macrophages [11]. Collectively, our results indicate the significance of alternatively activated macrophages in the inflammation seen in PM/DM-related ILD.

Serum sCD163 levels were significantly higher in patients with PM/DM-related ILD than those in healthy volunteers, which is consistent with the results of the recent study by Peng et al. [13]. They measured serum sCD163 levels in 108 patients with PM/DM, consisted of 65 patients without ILD and 43 patients with ILD, and found that serum sCD163 levels were correlated with the activity of myositis. However, the clinical implication of sCD163 in PM/DM-related ILD remains unclear. In the present study, we focused on the ILD and further advanced the understandings of sCD163 physiology in this disease entity, particularly the relevance to pulmonary function and prognosis.

In the Cox proportional hazard modeling, higher serum sCD163 was significantly associated with worse prognosis. This result implies that macrophage activation in PM/DM-related ILD plays an important role in advancing the disease. In contrast, several studies have evaluated the clinical significance of serum ferritin, which is a key biomarker of MAS, in PM/DM-related ILD [27-29]. Indeed, PM/DM is known to cause MAS, in which the lack of $\mathrm{T}$ cell regulation and excessive production of cytokines, including TNF- $\alpha$, IL-1 $\beta$, IL- 6 , and IL-18, results in macrophage activation. Those previous studies reported that hyperferritinemia was associated with high mortality in patients with PM/DM-related (particularly CADM-related) ILD, which was reproduced in our cohort. Interestingly, there was a weak, but not significant correlation between serum sCD163 levels and ferritin levels in the present study. The total value of serum ferritin might be affected by the storage of iron (in the form of L-subunit ferritin). Meanwhile, sCD163 is secreted from activated macrophages and elevated for days in peripheral blood, suggesting that this molecule specifically reflects the degree of macrophage activation. Although the superiority of either of the two biomarkers could not be evaluated in our small cohort, our results suggest that serum sCD163 may have value in the assessment of disease severity in PM/DM-related ILD.

Human CD163 expression has been confirmed in several tissues, such as red pulp macrophages, bone marrow 


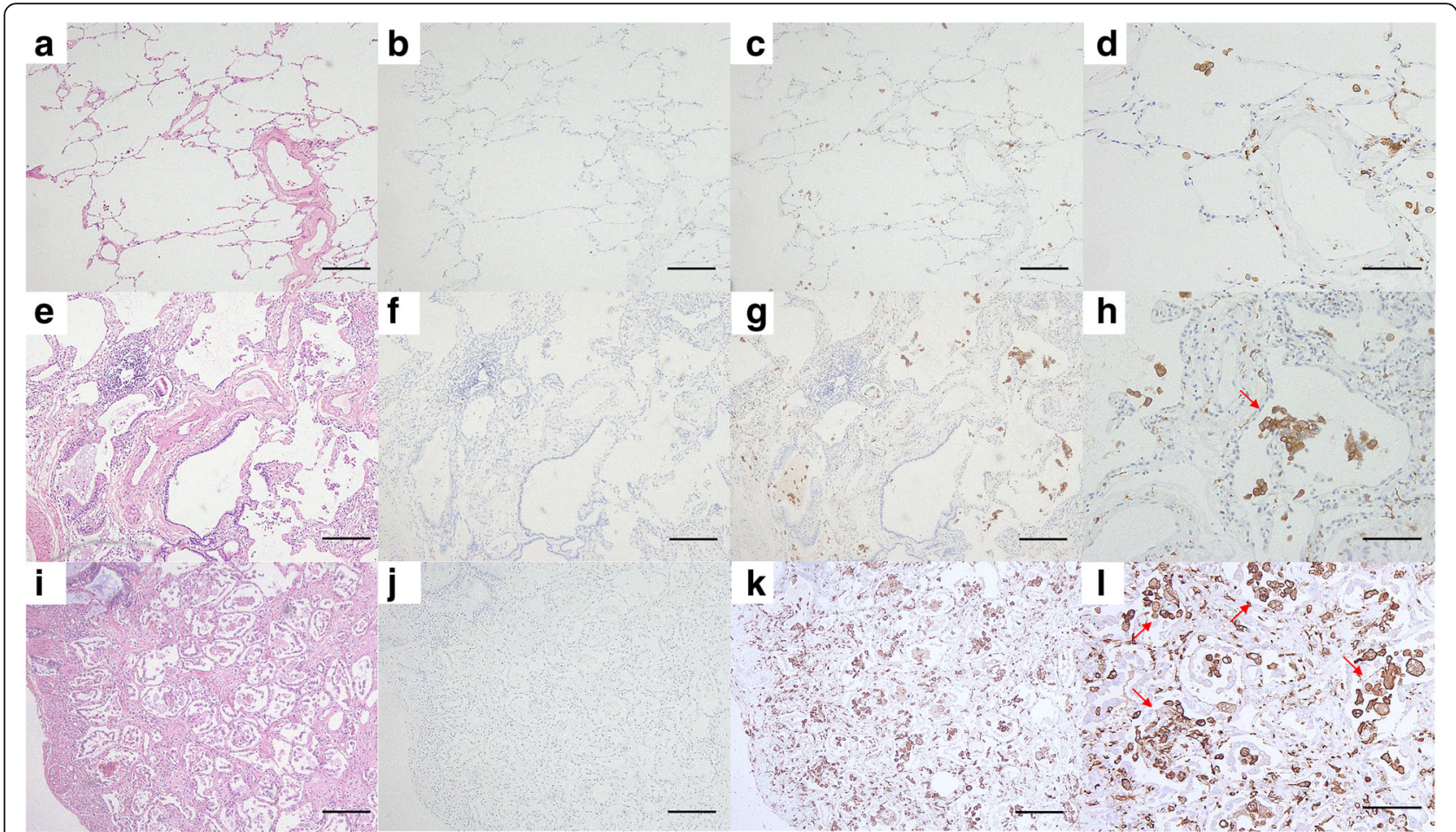

Fig. 3 Immunohistochemical staining. Lung sections from an early lung cancer patient (a-d), a surviving patient with classic dermatomyositis (DM)-related interstitial lung disease (ILD) (e-h: 66 year-old male patient, serum sCD163 $=519 \mathrm{ng} / \mathrm{mL}$ ), and a non-surviving patient with classic DM-related ILD (i-I: 32 year-old male patient, serum sCD163 $=1572 \mathrm{ng} / \mathrm{mL}$ ) are shown. CD163-positive macrophages are evident in the lungs of the two patients with classic DM-related ILD, especially in lung of the non-survivor. Accumulations of CD163-positive alveolar macrophages are observed (arrows). a, e, and i Hematoxylin - eosin staining; scale bar $200 \mu \mathrm{m}$. b, f, and j jsotype controls, scale bar $200 \mu \mathrm{m}$. c, g, and $\mathbf{k}$ Staining with anti-CD163 antibody with lower magnification (×40), scale bar $200 \mu \mathbf{m}$. d, h, and I Staining with anti-CD163 antibody with higher magnification $(\times 100)$, scale bar $100 \mu \mathrm{m}$

macrophages, liver macrophages (Kupffer cells), and alveolar macrophages $[10,11]$. In fact, on the basis of immunohistochemical analysis, one of the sources of elevated serum SCD163 seemed to be the diseased lungs. Additionally, more severe infiltration of CD163-positive macrophages was found in the lung of the non-surviving patient with DM-related ILD, compared with that in the lung of the survivor (Fig. 3). Although we are speculating on the assessment of only two lung specimens, the amount of infiltrating CD163-positive macrophage may reflect the severity of PM/DM-related ILD. The following results may support this: serum sCD163 was negatively correlated with percent predicted FVC. Previous studies demonstrate that alveolar macrophages produce several profibrotic cytokines including transforming growth factor- $\beta$, platelet-derived growth factor, and fibroblast growth factor-2 [16, 30, 31]. Intriguingly, Gibbons et al. recently demonstrated the paradoxical and essential role played by macrophages in the pathogenesis of lung fibrosis, in which activated macrophages had both profibrotic and resolution-promoting roles during fibrogenesis and the reversible phase, respectively [17]. Thus, the exact functions of CD163-positive macrophages in the pathogenesis of PM/DM-related ILD remain unclear, but the infiltration to the lungs may have some impact not only on inflammation but also on fibrosis.

The present study has several limitations. First, this was a small, retrospective study, which may have involved various biases. Validation in larger and prospective cohorts is needed to confirm our preliminary results. Second, there are several subtypes in the development of PM/DM-related ILD, such as ILD-preceding, concomitant, and myositis-preceding patterns, which indicates the difficulty in determining the exact ILD onset, particularly in patients with no symptoms or only minor symptoms. Our definition of ILD onset might be not accurate in some patients. Third, the patients included in the present study were not treated according to a consistent regimen. Although most of the patients were treated with corticosteroids and additional immunosuppressants, regimen variations, such as the doses and selection of drugs, may have affected the clinical disease course and prognosis. Additionally, we could not follow serum sCD163 levels chronologically. It is unclear how changes correlated with the clinical course of PM/DM- 
related ILD. In future studies, clinical factors important in the management of ILD, such as changes in pulmonary function and oxygenation, and treatment response, should be compared with changes in sCD163 levels. These would explore the usefulness of this biomarker.

\section{Conclusions}

Serum sCD163 might have potential for predicting the disease severity and prognosis in PM/DM-related ILD. Our results suggest the involvement of macrophage activation in this disease.

\section{Additional file}

Additional file 1: Table S1. Baseline characteristics of all subjects included in this study. Table S2. Comparison of baseline characteristics between patients with higher and lower values of serum soluble CD163 (cutoff $800 \mathrm{ng} / \mathrm{mL}$ ). (DOCX $45 \mathrm{~kb}$ )

\section{Abbreviations}

ARS: aminoacyl-tRNA synthetase; BAL: bronchoalveolar lavage; CADM: clinically amyopathic dermatomyositis; CK: creatine kinase; CRP: Creactive protein; DLCO: diffusing capacity of the lung for carbon monoxide; DM: dermatomyositis; FVC: forced vital capacity; HRCT: high-resolution computed tomography; IL: interleukin; ILD: interstitial lung disease; MAS: macrophage activation syndrome; MDA5: Melanoma differentiationassociated gene 5; PaO2: arterial partial pressure of oxygen; PM: polymyositis; sCD163: soluble CD163; TNF: tumor necrosis factor

\section{Acknowledgements}

We thank Dr Hajime Yamakage (SATISTA Inc.) and Dr Masaaki Kaneta (Department of Clinical Pharmacology and Therapeutics, Hamamatsu University School of Medicine) for the statistical advice and immunohistochemistry procedures, respectively.

\section{Funding}

Not applicable.

\section{Availability of data and materials}

The datasets used and/or analyzed during the present study are available from the corresponding author on reasonable request.

\section{Authors' contributions}

YE and YS contributed to study design, data acquisition, data interpretation, and drafting the manuscript. KM analyzed the data and revised the manuscript. $\mathrm{HH}, \mathrm{TF}, \mathrm{DS}$, and $\mathrm{NO}$ recruited patients and revised the manuscript. MKo, MKa KF, NE, YN, NI, RN, TM, TI, and TS contributed to data interpretation and revision of the manuscript. All authors read and approved the final manuscript.

\section{Competing interests}

The authors declare that they have no competing interests.

\section{Consent for publication}

Not applicable.

\section{Ethics approval and consent to participate}

This study was approved by the review board of Hamamatsu University School of Medicine in Japan (approved number 15-197). Given the retrospective nature of the study, written informed consent from patients was not required.

\section{Author details}

'Second Division, Department of Internal Medicine, Hamamatsu University School of Medicine, 1-20-1 Handayama, Hamamatsu, Shizuoka 431-3192, Japan. ${ }^{2}$ Department of Regenerative and Infectious Pathology, Hamamatsu
University School of Medicine, Hamamatsu, Japan. ${ }^{3}$ Department of Clinical Pharmacology and Therapeutics, Hamamatsu University School of Medicine, Hamamatsu, Japan. ${ }^{4}$ Third Division, Department of Internal Medicine, Hamamatsu University School of Medicine, Hamamatsu, Japan. ${ }^{5}$ Department of Rheumatology and Clinical Immunology, Kyoto University Graduate School of Medicine, Kyoto, Japan.

Received: 21 September 2016 Accepted: 28 December 2016

Published online: 19 January 2017

\section{References}

1. Ernste FC, Reed AM. Idiopathic inflammatory myopathies: current trends in pathogenesis, clinical features, and up-to-date treatment recommendations. Mayo Clin Proc. 2013;88:83-105.

2. Bohan A, Peter JB. Polymyositis and dermatomyositis (first of two parts). N Engl J Med. 1975;292:344-7.

3. Mathai SC, Danoff SK. Management of interstitial lung disease associated with connective tissue disease. BMJ. 2016;352:h6819.

4. Fujisawa T, Suda T, Nakamura Y, Enomoto N, Ide K, Toyoshima M, et al. Differences in clinical features and prognosis of interstitial lung diseases between polymyositis and dermatomyositis. J Rheumatol. 2005;32:58-64.

5. Suda T, Fujisawa T, Enomoto N, Nakamura $Y$, Inui N, Naito T, et al. Interstitial lung diseases associated with amyopathic dermatomyositis. Eur Respir J. 2006;28:1005-12.

6. Fujisawa T, Hozumi H, Kono M, Enomoto $N$, Hashimoto D, Nakamura $Y$, et al. Prognostic factors for myositis-associated interstitial lung disease. PLoS One. 2014;9:e98824.

7. Hozumi H, Enomoto N, Kono M, Fujisawa T, Inui N, Nakamura $Y$, et al. Prognostic significance of anti-aminoacyl-tRNA synthetase antibodies in polymyositis/dermatomyositis-associated interstitial lung disease: a retrospective case control study. PLoS One. 2015;10:e0120313.

8. Sasano H, Hagiwara E, Kitamura H, Enomoto $Y$, Matsuo N, Baba T, et al. Long-term clinical course of anti-glycyl tRNA synthetase (anti-EJ) antibodyrelated interstitial lung disease pathologically proven by surgical lung biopsy. BMC Pulm Med. 2016:16:168.

9. Fabriek BO, Dijkstra CD, van den Berg TK. The macrophage scavenger receptor CD163. Immunobiology. 2005:210:153-60.

10. Lau SK, Chu PG, Weiss LM. CD163: a specific marker of macrophages in paraffin-embedded tissue samples. Am J Clin Pathol. 2004;122:794-801.

11. Etzerodt A, Moestrup SK. CD163 and inflammation: biological, diagnostic, and therapeutic aspects. Antioxid Redox Signal. 2013;18:2352-63.

12. Dorph C, Englund P, Nennesmo I, Lundberg IE. Signs of inflammation in both symptomatic and asymptomatic muscles from patients with polymyositis and dermatomyositis. Ann Rheum Dis. 2006;65:1565-71.

13. Peng QL, Zhang YL, Shu XM, Yang HB, Zhang L, Chen F, et al. Elevated serum levels of soluble CD163 in polymyositis and dermatomyositis: associated with macrophage infiltration in muscle tissue. J Rheumatol. 2015:42:979-87.

14. Dalakas MC, Hohlfeld R. Polymyositis and dermatomyositis. Lancet. 2003:362:971-82.

15. Dalakas MC. Mechanisms of disease: signaling pathways and immunobiology of inflammatory myopathies. Nat Clin Pract Rheumatol. 2006;2:219-27.

16. Khalil N, Bereznay O, Sporn M, Greenberg AH. Macrophage production of transforming growth factor beta and fibroblast collagen synthesis in chronic pulmonary inflammation. J Exp Med. 1989;170:727-37.

17. Gibbons MA, MacKinnon AC, Ramachandran P, Dhaliwal K, Duffin R Phythian-Adams AT, et al. Ly6Chi monocytes direct alternatively activated profibrotic macrophage regulation of lung fibrosis. Am J Respir Crit Care Med. 2011;184:569-81.

18. Møller HJ, Moestrup SK, Weis N, Wejse C, Nielsen H, Pedersen SS, et al. Macrophage serum markers in pneumococcal bacteremia: prediction of survival by soluble CD163. Crit Care Med. 2006;34:2561-6.

19. Suzuki Y, Enomoto Y, Yokomura K, Hozumi H, Kono M, Karayama M, et al. Soluble hemoglobin scavenger receptor CD163 (sCD163) predicts mortality of community-acquired pneumonia. J Infect. 2016;73:375-7.

20. Kusi KA, Gyan BA, Goka BQ, Dodoo D, Obeng-Adjei G, Troye-Blomberg M, et al. Levels of soluble CD163 and severity of malaria in children in Ghana. Clin Vaccine Immunol. 2008;15:1456-60.

21. Greisen SR, Moller HJ, Stengaard-Pedersen K, Hetland ML, Hørslev-Petersen K, Jørgensen A, et al. Soluble macrophage-derived CD163 is a marker of 
disease activity and progression in early rheumatoid arthritis. Clin Exp Rheumatol. 2011;29:689-92.

22. Nakayama W, Jinnin M, Makino K, Kajihara I, Makino T, Fukushima S, et al. Serum levels of soluble CD163 in patients with systemic sclerosis. Rheumatol Int. 2012;32:403-7.

23. Suzuki Y, Shirai M, Asada K, Miwa S, Karayama M, Nakamura Y, et al. Utility of macrophage activated marker, CD163, for diagnosis and prognosis in pulmonary tuberculosis. Ann Am Thorac Soc. 2017;14(1):57-64.

24. Euwer RL, Sontheimer RD. Amyopathic dermatomyositis: a review. J Invest Dermatol. 1993;100:124S-7S.

25. Goh NS, Desai SR, Veeraraghavan S, Hansell DM, Copley SJ, Maher TM, et al. Interstitial lung disease in systemic sclerosis: a simple staging system. Am J Respir Crit Care Med. 2008;177:1248-54.

26. Heagerty PJ, Zheng Y. Survival model predictive accuracy and ROC curves. Biometrics. 2005;61:92-105.

27. Gono T, Sato S, Kawaguchi Y, Kuwana M, Hanaoka M, Katsumata Y, et al. Anti-MDA5 antibody, ferritin and IL-18 are useful for the evaluation of response to treatment in interstitial lung disease with anti-MDA5 antibodypositive dermatomyositis. Rheumatology (Oxford). 2012;51:1563-70.

28. Zou J, Guo Q, Chi J, Wu H, Bao C. HRCT score and serum ferritin level are factors associated to the 1-year mortality of acute interstitial lung disease in clinically amyopathic dermatomyositis patients. Clin Rheumatol. 2015;34:707-14.

29. Isoda K, Takeuchi T, Kotani T, Hata K, Shoda T, Ishida T, et al. Pre-treatment ferritin level and alveolar-arterial oxygen gradient can predict mortality rate due to acute/subacute interstitial pneumonia in dermatomyositis treated by cyclosporine a/glucocorticosteroid combination therapy: a case control study [corrected]. PLoS One. 2014;9:e89610.

30. Bonner JC. Regulation of PDGF and its receptors in fibrotic diseases. Cytokine Growth Factor Rev. 2004;15:255-73.

31. Henke C, Marineili W, Jessurun J, Fox J, Harms D, Peterson M, et al. Macrophage production of basic fibroblast growth factor in the fibroproliferative disorder of alveolar fibrosis after lung injury. Am J Pathol. 1993;143:1189-99.

\section{Submit your next manuscript to BioMed Central and we will help you at every step:}

- We accept pre-submission inquiries

- Our selector tool helps you to find the most relevant journal

- We provide round the clock customer support

- Convenient online submission

- Thorough peer review

- Inclusion in PubMed and all major indexing services

- Maximum visibility for your research

Submit your manuscript at www.biomedcentral.com/submit

) Biomed Central 